\title{
Einiges über verschiedene Guttaperchasorten mit besonderer Berücksichtigung der Tjipetir-Guttapercha.
}

\author{
Von Rudolf Ditmar.
}

(Eingegangen an 6. Marz 1012)

(Aus dem Kautschukiaboratorium Dr. Rudolf Ditmar, Graz.)

Guttapercha, welche von den Malaien "Getah pertja " genannt wird, wurde in Europa im Jahre 1843 bekannt. In der Industrie findet Guttapercha hauptsächlich zur Kabelisolation Verwendung. Die gesamte Lünge aller unterseeischen Telegraphen-Kabel beträgt ungefähr $439047 \mathrm{~km}$. Dafür sind allein ca. $20594000 \mathrm{~kg}$ Guttapercha erforderlich. Hierzu kommen noch die unterirdischen Kabel, über welche genaue Zahlenangaben fehlen.

1883 erhielt W. Burck von der holländischen Regierung den Auftrag, eine Forschungsreise zum Studium der Guttaperchabäume anzutreten, da man fürchtete, daB die Guttaperchabäume durch den Raubbau, der von den Einheimischen getrieben wurde, auf den Aussterbeetat gesetzt seien. W. Bu r ck gebührt das Verdienst, die Baume festgestellt $z u$ haben, aus welchen die verschiedenen Guttaperchasorten gewonnen werden. Aus seinen Untersuchungen geht hervor, daßdie für die Katelindustrie verwertbare Guttapercha nur von wenigen Baumgattungen gewornen wird, welche immer seltener in den Urwäldern vorkommen. Nach W. Burck sind es Bäume, weiche zur Familie der Sapotazeen gehören, nămlich: die Gattung Palaquium, von welcher Pal. oblongifolium, Pal. borneensis und Pal. gutta die beste Guttapercha liefern.

Diese Bäume kommen auf einem kleinen Gebiete vor, welches zwischen dem sechsten Grade nördlicher und südlicher Breite und dem 99. und 119. östlichen Längengrade liegt. Der gröBte Teil dieses Gebietes ist in NiederlandischIndien, wo auch die größte Menge Guttapercha gewonnen wird.

Die Größe des Verbrauches an Guttapercha wird erst recht verständlich, wenn einmal die heute im Betriebe liegenden unterseeischen Kabel erneuert werden müssen. Für einen Kilometer Kabel sind ungefähr $25 \mathrm{~kg}$ Guttapercha erforderlich, ein 15-20jähriger gefällter Baum liefert nicht mehr als $400 \mathrm{~g}$ Guttapercha. Dieses Moment zog Cremer, ein Minister der holländischen Kolonien, in Erwägung und beschlob auf den Rat von Professor M. Treub, dem Direktor der botanischen Cärten von Buitenzorg, die Förderung der Kultur der Guttaperchabäume. 1900 sollte eine königliche Gutta- perchaplantage unter der Leitung $M$. Treub's errichtet werden. Als der geeignetste Ort dafür erwies sich Tjipetir auf java.

Die königliche Plantage in Tjipetir liegt in der Residenz Preanger Regencies, acht Meilen von der Eisenbahnstation Tjibadak, 17 Meilen von der Wynkoops-Bai entfernt an der Südküste Javas; sie liegt auf einer Anhöhe, ca. 1700 Meter über dem Meere. Unter den Samen tragenden Bäumen von Tjipetir ist die Gattung Palaquium oblongifolium am hăufigsten anzutreffen. Palaquium Gutta, Pal. oblongifolium und Pal, borneense haben groBe Aehnlichkeit miteinander und geben alle ein vortreffliches Produkt. Auf den königlichen Guttaperchaplantagen werden jetzt ausschlieblich diese drei Gattungen gezogen. Der Flächenraum der Guttaperchaplantage von Tjipetir beläuft sich auf ca. 2500 Morgen. Die Guttapercha findet sich nicht nur in der Rinde, sondern auch in den Blättern. Die Rinde enthält ungefähr 5 Proz. Guttapercha, wovon 3 Proz. Gutta sind. Die frischen Blätter der besseren Arten enthalten ca. 10 Proz. Guttapercha mit 5 Proz. Gutta auf Trockensubstanz berechnet. Die frische Rinde enthält ca. 65 Proz. Wasser, frisch gepflückte Blätter enthalten 60 Proz. Rinde und Blätter von ganz jungen Bäumen enthalten mehr Wasser. Ein Teil des Latex wird in der einfachsten Weise durch Einschnitte aus der Rinde gewonnen. Schwieriger ist die Gewinnung der Guttapercha aus den Blättern. Zuerst versuchte man eine Extraktion der Blätter mit Kohlenwasserstoffen, wiz: z. B. Toluol, Benzin usw. Die Resultate waren nicht günstig, weil die Guttapercha durch Behandlung mit diesen Stoffen Veränderungen erleidet, welche sie als Isoliermasse unbrauchbar machen. Durch eine mechanische Operation erhielt man bessere Ergebnisse. Arnaud und später Lederboer führten eine Methode ein, die darin besteht. daß die frischen Blätter zu einem Brei zermahlen werden, der dann mit Wasser ausgekocht wird. An der Oberfläche scheidet sich die Guttapercha ab. Tromp de Haas gelang es auch, aus alten abgefallenen Blättern auf mechanische Weise Guttapercha abzuscheiden. Das Erträgnis ist dabei allerdings nicht so groß wie bei frischen 
Blättern. Die Gewinnung aus den Blättern hat folgende Vorteile:

1. Bei den Plantagenbäumen kann man schon in diritten oder vierten Jahre den Versuch maclien, die Guttapereha aus den Blättern $z u$ gewinnen, wngegen man den Stamm nicht vor 15 Jahren anzapfen kann.

2. Auf diese Weise wird die größte Ertragsfähigkeit erzielt.

3. Diese Gewinnungsart ist dem Baume am wenigsten schädlich. Die Produktion an Guttapercha aus den Blättern beläuft sich auf der ganzen Plantage Tjipetir im Jahre 1912 auf ca. $2780000 \mathrm{~kg}$.

Die Samenkörner der Guttaperchabăume enthalten auch ein vegetabilisches Fett, welches sich sehr gut für verschiedene technische $Z$ wecke verwenden läBt. Der Schmelzpunkt des Fettes liegt bei ungefahr $40^{\circ} \mathrm{C}$, bei gewöhnlicher Temperatur ist es fest. Es besteht aus:

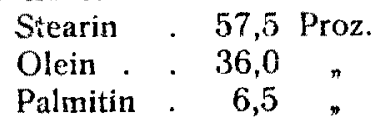

Im Jahre 1906 trugen von 8596 zehnjährigen Guttaperchabäumen nur 2360 Stïck Früchte. Daraus hătte man $1850 \mathrm{~kg}$ Fett gewinnen können.

Die Tipetir-Guttapercha, wie sie Tromp de $\mathrm{H}$ a a s auf Java erzeugt, kommt in Platten von ca. $30 \mathrm{~cm}$ Breite, $45 \mathrm{~cm}$ Länge und $24 \mathrm{~mm}$ Höhe in den Handel. Sie hat ein hellbraungrünliches Aussehen und ist so ausgezeichnet rein, dal man sich das Waschen derselben in der Gummi- oder Kabelfabrik ersparen kann. Das Kilo stellt sich ungefähr auf $9 \frac{1 / 2}{2}$ Mark. Auf den Platten ist in dirken Lettern der Name "Tjipetir" eingraviert. Die Anulyse einer solchen Platte ergab:

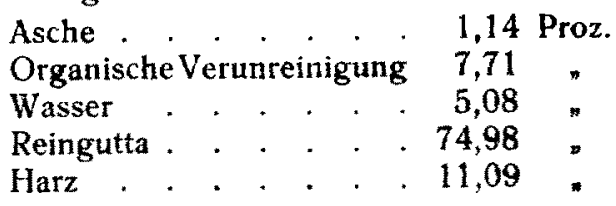

Es hat sich herausgestelit, dab man Harze aus der Guttaperchs mit Azeton mur sehr schwer herauslösen kann, da Axcton sehr wenig in die Tiefe der feingeschnittenen Guttapereha dringt und infolgedessen das Harz nicht quantitativ extrahiert wind. Wärend nan bein Kautschuk in den meisten Untenuchungsantalten noch am Azeton festhält, halte ich es bei der Guttapercha für sehr zweckmäßig die Harzextraktion anstatt mit Azeton mit Essigather vorzunehmen. Das mit Essigäther extrahiette Harz der TjipetirGuttapercha hat ein schönes grünliches Aus- sehen und ist wesentlich verschieden von den Harzen anderer Guttaperchasorten. Zum Zwecke der Esssigätherextraktion schneidet man die Guttapercha in möglichst kleine Stückchen mit einem scharfen Messer. Ein Einlegen dieser Guttaperchastückchen in eine Soxhlethülse ist unzweckmäßig, da die Guttapercha bei der Extraktion weich wird und an der Hülse anklebt. Das Wägen der Hülse mit der Guttaporcha ist immer umständlich und ungenau, ta das Papier der Hülse hygroskopisch ist. Aluminiumhülsen versagen bei der Guttapercha vollständig, indem die Löcher von der weichen Guttapercha verstopft werden. Das Wägen des vom Extraktionsmittel befreiten Harzes ist nicht immer sicher. Gewisse Harzanteile der Guttapercha nehmen nämlich beim Trocknen durch Oxydation stark zu. Die Soxhletkolben lassen sich schwer im Vakuum trocknen, leichter geht dies mit der Guttapercha. Immerhin ist eine Kontrolle durch Wägen des Harzes aus der extrahierten Guttapercha stets am Platze. Es hat sich als vorteilhaft erwiesen, die Harzextraktion bei Guttapercha mit Essigäther nicht im Soxhletapparate, sondern in einem Soxhletkolben mit aufgesetztem Rückflußkühler direkt vorzunehmen. Man extrahiert am besten unter dreimaligem Wechseln des Essigäthers einen ganzen Tag. Alle Guttaperchaharze sind losslich in Essigäther, während der Guttakohlenwasserstoff unlöslich ist.

A. Tschirch gibt in seinem Werke "Die Harze und die Harzbehäler" an, daß die Harze der Guttapercha aus Albanen, Albananen und Fluavile bestehen. Albane sind nach A.T schirch nur in siedendem Alkohot löslich, Fluavile schon im kaiten, Albanane weder im siedenden noch im kalten Alkohol. A. Tschirch meint, daB das Alban die guten Eigenschaften der Guttapercha nicht beeintrüchtigt, vielleicht soger für eine gute Handelsware notwendig int; er behouptet ferner, daB das Fluavil, sobald es in betridchtlicher Menge auftritt, den Wert der Guttapercha herabsetzt. Wie wir später aus unseren Untersuchungen seken werden, ist diese Ansicht A. Tschirch's nicht allgemein gültig.

Eine Reihe von Hondelsguttaperchasorten und zwar Tilpetir, weike Ceylon-PlantagenGuttapercha, Gulai prima, Gulei sekunda, Akassa, Siak und Penang wurden eingehendst untersucht und eine technische Trennung der angefübrten drei Harzsorten im Gesawtharze durchgeführt. Ich verdanke die Guttaperchasorten der Liebenswürdigkeit des Hern E war $\$ B$ arbary von den Roskear Fuse Works in Camborne. 
Die Trennung der einzelnen Harzsorten im Gesamtharz der Guttapercha wird in folgender Weise durchgeführt. Der im Vakuum oder Trockenschrank bei $100-103^{\circ}$ getrocknete Essigătherextrakt (die Trocknung im Trockenschrank ist nicht immer zulässig, wenn die Harze oxydieren) wird zwei Stunden in der Schüttelmaschine mit kaltem Alkohol ausgeschüttelt. Dadurch geht das Pluavil in Lösung, wăhrend das Alban und Albanan zurückbleibt. Der Alkohol wird vom Fluavil abdestilliert, das Fluavil entweder, wenn angängig, bei $100-103^{\circ}$, sonst im Vakuum über Paraffin bei mäbiger Wärme zur Gewichtskonstanz getrocknet und gewogen. Der Rückstand besteht aus Alban und Albanan und wird mit Alkohol gekocht, dabei gehen die Albane in Lösung, der Rückstand ist Albanan. Die Albane werden nun in derselben Weise behandelt, wie oben das Fluavil, und gewogen. Der Albanrückstand unterliegt der gleichen Behandlung. Wir haben also folgendes Schema:

$$
\begin{aligned}
& \underbrace{\text { Alter Alkohol löst Fluavil }}_{\text {Alban + Albanan + Fluavil }} \\
& \frac{\text { Alban + Albanan }}{\text { + heiler Alkohol löst Alban }} \\
& \text { Albanan (oxydierk nicht). }
\end{aligned}
$$

Die Wasserbestimmung in der Gutrapercha geschieht durch Trocknen des dünnen Pelks im Vakuum bei $60^{\circ}$ über Chlorkalzium bis zur Gewichtskonstanz.

Der organische Schmutz wird in der Weise bestimmt, daf $0,2-0,3 \mathrm{~g}$ Guttapercha in $100 \mathrm{ccm}$ Chloroform oder Benzol gelöst werden. Dann wird in einer Zentrifuge mit hoher Tourenzahl vier- bis fünfmal auszentrifugiert, bis die Losung absolut rein ist. Dann schüttelt man den Schmutz, der aus anorganischen und organischen Bestandteilen besteht, mehrere Male mit Benzol aus und entrifugiert ihn aufs neue. Die Operation wird so lange wiederholt, bis man sicher ist, daB keine Guttapercha und kein Harz mehr im Schmutx zurückblcibt. Hernach schüttelt man den Sehmetz wieder in Benzol und filtriert ihn durch eimen Platin-Goochtiegel oder einen gewöhnlicten Goochticgel, trocknet bei $103^{\circ}$ und wiegt. Man erhält organischen und anorganischen Schmutz. Verascht man, dann verbrennt der organiache Schmutz und aus der Differens berechnet man den anorganischen. Die Arche kann auch durch dirckte Veraschung bestimmt werden. Dic Resultate der sieben untessuchten Guttaperchasorten sind it: nachfolgender Tabelle $I$ und $I$ angeführt.

Auber diesen Bestimmungen wurden an den skben Guttaperchasorten auch noch Viskosität und Exweichungspunkt festgestellt.

\begin{tabular}{|c|c|c|c|c|c|c|c|c|}
\hline $\begin{array}{l}\text { Name der } \\
\text { Guttapercha }\end{array}$ & \begin{tabular}{|c|}
$\begin{array}{c}\text { gewasehen } \\
\text { oder } \\
\text { ungewasehen }\end{array}$ \\
\end{tabular} & $\begin{array}{l}\text { Harz } \\
\text { Proz. }\end{array}$ & $\begin{array}{l}\text { Westex } \\
\text { Proz. } \\
\end{array}$ & $\begin{array}{c}\text { org. Schmmz } \\
\text { Proz. }\end{array}$ & $\begin{array}{l}\text { anorg. } \\
\text { Schrnutz } \\
\text { Proz. } \\
\end{array}$ & Oute & $\begin{array}{l}\text { Preise in } \\
\text { Mark ca. }\end{array}$ & Anmerkungen \\
\hline Tjipetir & ungewaschen & 11,09 & 5,08 & 7,71 & 1,14 & 74,98 & $\cdot 9.17$ & $\begin{array}{l}\text { aus Błattern extrahiert, } \\
\text { Harz ist grasgriun }\end{array}$ \\
\hline $\begin{array}{c}\text { WeiBe } \\
\text { Outtapercha }\end{array}$ & gewaschen & 48,65 & $\begin{array}{c}\text { kein } \\
\text { Wasser }\end{array}$ & 1,09 & 0,20 & 49,10 & $?$ & $\begin{array}{l}\text { ist gebleicht. Hat } \\
\text { beim Trocknen um ca. } \\
1,6 \text { Proz. zugenommen }\end{array}$ \\
\hline Gulal prima & gewaschen & 48,58 & 15,27 & 1,52 & 0,87 & 33,76 & 5.51 & \\
\hline $\begin{array}{c}\text { Gulai } \\
\text { sekunda }\end{array}$ & gewaschen & 55,39 & 12,67 & 1,48 & 0,92 & 29,54 & 5.09 & \\
\hline Akassa & gewaschen & 73,19 & 11,46 & 2,54 & 0,70 & 12,83 & 2.38 & \\
\hline Siak & gewaschen & 69,50 & 11,31 & 3,54 & 1,31 & 14,34 & 2.20 & $\begin{array}{c}\text { Hat beim 2weiten } \\
\text { Trocknen in Vakuum } \\
\text { zugenommen } \\
\end{array}$ \\
\hline Penang & gewaschen & 65,22 & 9,84 & 3,72 & 0,81 & 20,41 & 2.55 & \\
\hline
\end{tabular}

Die Viskositätsbestimmungen wurden in einem C. Engler'schen Viskosimeter durchgefahrt, welcher für die Guttapercha vollständig

Tabelle I 
Tabelle II

Harztrennugen.

\begin{tabular}{c|c|c|c|c|c|c}
\hline Guttaperchasorte & $\begin{array}{c}\text { Gesamtharze } \\
\text { Proz. }\end{array}$ & $\begin{array}{c}\text { Fluavil } \\
\text { kalter Alkohol } \\
\text { Proz. }\end{array}$ & $\begin{array}{c}\text { Alban } \\
\text { heiBer Alkohol } \\
\text { Proz. }\end{array}$ & $\begin{array}{c}\text { Albanane } \\
\text { Proz. }\end{array}$ & Anmerkungen \\
\hline Tijpetir & 11,09 & 5,84 & 4,72 & 0,53 & $\begin{array}{c}\text { Fluavile oxydierten, sind aus } \\
\text { der Differenz gerechnet }\end{array}$ \\
\hline $\begin{array}{c}\text { WeiBe } \\
\text { Guttapercha }\end{array}$ & 48,65 & 39,36 & 9,26 & 0,03 & $\begin{array}{c}\text { Fluavile oxydierten, sind aus } \\
\text { der Differenz gerechnet }\end{array}$ \\
\hline Gulai prima & 48,58 & 27,55 & 20,55 & 0,48 & \\
\hline Gulai sekunda & 55,39 & 21,07 & 32,45 & 1,87 & \\
\hline Akassa & 73,19 & 10,54 & 62,45 & 0,20 & Albane aus der Differenz ge- \\
\hline Siak & 69,50 & 14,58 & 49,00 & 5,92 & \\
\hline Penang & 65,22 & 18,49 & 42,89 & 3,84 & \\
\hline
\end{tabular}

genügt und ganz scharfe Resultate gibt. Es ist ganz überflüssig, sich dabei des Viskosimeters von P. Altm a nn in Berlin für Kautschuklösungen $\mathrm{zu}$ bedienen. Ueberhaupt möchte ich alle Komplikationen bei derartigen Bestimmungen vermeiden, welche wieder auf die Herstellung spezieiler Apparate ausgehen, wenn es nicht unbedingt notwendig ist. Und hier ist es nicht unbedingt notwendig, weil das $C$. Engler'sche Viskosimeter die gleichen Dienste leistet und anch bei der Bestimmung der Oele verwendet wird. Die Viskositäten wurden stets dreimal bei jeder Guttaperchasorte durchgeführt und ergaben immer dasselbe Resultat. ZurViskositätsbestimmung von Kautschuklösungen ist das Viskosimeter von P. Altmann dem C. Englerschen entschieden vorzuziehen. Kautschuklösungen sind auch in ihrem Verhalten wesentlich verschieden von Guttaperchalösungen. Zur Viskositätsbestimmung wurden $2 \mathrm{~g}$ Guttapercha in $100 \mathrm{ccm}$ Tetrachlorkohlenstoff in einer Schüttelmaschine zirka zwei Stunden geschüttelt, bis vollkommene Lösung eingetreten ist. Alle Viskositätsbestimmungen wurden bei $15^{\circ} \mathrm{C}$ durchgeführt: Die Viskositäten beziehen sich nicht auf Wasser, sondern auf Tetrachlorkohlenstoff als auf das verwendete Lösungsmittel.

Neben der Viskosität wurde auch der Erweichungspunkt der einzelnen Guttaperchasorten bestimmt. Dieser wurde in einer etwas anderen
Art durchgeführt als es $\mathrm{E} . \mathrm{Ob}$ a ch angibt. Die Methode von $\mathrm{E} . \mathrm{Ob}$ a ch besteht darin, daB man Guttaperchastreifen wie in einem Zerreißapparate zwischen zwei Backen einklemmt, von denen die eine von einer Spiralfeder gezogen wird, während die andere in einem Rahmen festgehalten wird. Der ganze Apparat wird in ein Wasserbad eingesenkt, welches man langsam erwärmt. Sobald die Guttapercha zu erweichen beginnt, wird der Guttaperchastreifen durch die Zugkraft der Feder ausgezogen. Der ganze Apparat ist mit einem Läutewerk in Verbindung, welches sofort in Tätigkeit gesetzt wird, sobald der Streifen zu erweichen beginnt. Diese Methode hat sich im allgemeinen nicht recht in der Praxis bewährt. Der Grund liegt darin, daß bei beginnender Erweichung die Guttapercha aus den Klemmbacken herausgerissen wird. Auberdem lassen sich gewisse minderwertige Guttaperchasorten überhaupt nicht in den Apparat einklemmen, weil, sie bröckelig sind und schon früher zerreifien, bevor noch Erweichung eingetreten ist. Ich habe zuerst versucht, den Guttaperchastreifen durch ausgestanzte Guttapercharinge und die Einklemmung durch Rollen zu ersetzen. Auch dies hat sich in der Praxis nicht bewährt. Schließlich bin ich von dieser Methode ganz abgekommen und habe sie durch eine neue ersetzt, welche darin besteht, dak man einen stärkeren Guttaperchastreifen von 
$7 \mathrm{~cm}$ Länge und einem Querschnitt von $5 \mathrm{~mm}$ im Quadrat auf einer Seite $1 \mathrm{~cm}$ weit in einer Klemme festklemmt, während man am anderen Ende ein Gewicht von $2 \mathrm{~g}$ mittels einer Schraube befestigt. Der ganze Apparat wird in eine heizbare Wanne unter Wasser gestellt. In das Wasser wird ein Thermometer eingesenkt und das
Wasser wird langsam erwärmt. Sobald die Erweichung beginnt, krümmt sich der Guttaperchastreifen infolge der Schwere des Gewichtes und die Temperatur wird abgelesen. Dieser Apparat gibt die genauesten Resultate. Er läbi sich ebenso wie der $\mathrm{Obach}$ 'sche mit einem Läutewerk in Verbindung setzen.

Tabelle III

Zusammenstellung nach der Qualität der Guttaperchasorten in der Praxis.

\begin{tabular}{|c|c|c|c|}
\hline Sorte & $\begin{array}{c}\text { Gutta }\left(\mathrm{C}_{10} \mathrm{H}_{16}\right) \\
\text { Proz. } \\
\end{array}$ & Viskositat bei $15^{\circ} \mathrm{C}$ & Erweichungspunkt \\
\hline Tlipetir & 74,98 & 1,60 & 60 \\
\hline WeiBe Guttapercha & 49,10 & 1,44 & 50 \\
\hline Gulai prima & 33,76 & $1 \overline{1,59}$ & 47 \\
\hline Gulai sekunda & 29,54 & 1,33 & 37 \\
\hline Penang & 20,41 & 1,41 & 42 \\
\hline Akassa & 12,83 & 1,02 & 35 \\
\hline Siak & 14,34 & 1,12 & 40 \\
\hline
\end{tabular}

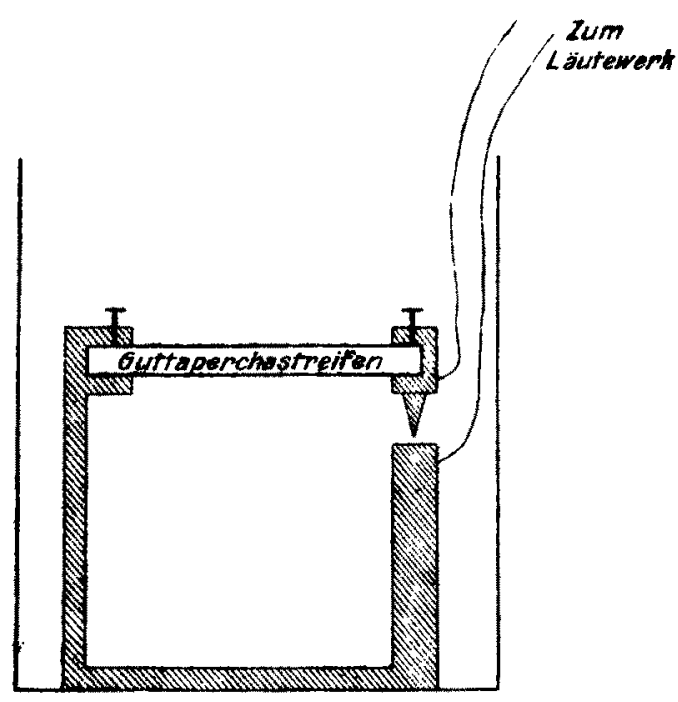

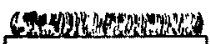

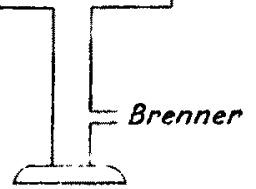

Aus Tabelle I und III ersehen wir, daf sich der Handelspreis im allgemeinen ziemlich genau nach dem Gehalte der Sorte an Reinguttakohlenwasserstoff richtet. Nur Akassa und Siak machen Ausnahmen, die Preisdifferenz ist aber hier nicht so grob.
Die Viskosităt steht in keinem Zusammenhang mit dem Preise, ebenso ist kein Zusammenhang zwischen Erweichungspunkt und Viskosität ersichtlich.

Nach A. Tschirch soll Fluavil den Wert der Handelsware herabsetzen. Aus Tabelle II ist dagegen ersichtlich, daB diese Behauptung völlig irrig ist. Die weibe Guttapercha stellt eine besonders wertvolle Guttaperchasorte vor und enthält 39,36 Proz. Fluavil, dasselbe gilt von Gulai prima, Gulai sekunda usw. Die von mir angeführten Preise beziehen sich auf die ungewaschenen Guttaperchasorten. Auch die Tschirch'sche Anschauung, daß Albane die guten Eigenschaften der Guttapercha nicht beeinträchtigen, ist unrichtig. Es zeig $\mathrm{sich}$ geradeim Gegenteil, dabein hoherer Gehalt an Alban und Albanan den Handelswert der Guttapercha im allgemeinen herabsetzt. Dennoch hat ein gleich mäBiges Fallen des Gehaltes an Alban und Albanan nicht eine $\mathrm{g} l \mathrm{l}$ i $\mathrm{ch}$ mä $\mathrm{B}$ ig e Preisverringerung zur Folge.

Frweichungspunkt und Gesamtharzgehalt stehen in einem gewissen Verhälnis zuein. ander, aber auch nur ganz allgemein. Je größer im allgemeinen der Gesantharzgehalt wird, desto tiefer liegt der Erweichungspunkt. Natürlıch spielt die Beschaffenheit uder sagen wir besser die Polymerisationsstufe des Reinguttakohlenwasserstoffe; auch eine grobic Rolle in bezug auf den Erweichungspunkt. Gesamtharz- 
gehalt und Polymerisationsstufe des Reinguttakohlenwasserstoffes sind neben anderen Faktoren die Komponenten, welche als Resultierende den Erweichungspunkt geben.

Für die Praxis spielen die Viskositätsbestimmungen bis heute fast gar keine Rolle. Der Praktiker kann daraus keine Schlüsse auf seine Ware ziehen, infolgedessen hat er keine Ursache Viskositätsbestimmungen auszuführen. Fragen wir uns, warum dem so ist. Der Grund liegt darin, daB sich an dem Rohmaterial, also an der ungewaschenen Guttapercha, keine Viskositätsbestimmungen ausführen lassen. Die Verunreinigungen organischer und anorganischer Natur würden das AusfluBrohr des Viskosimeters verstopfen. Macht man hingegen die Viskositätsbestimmungen an der gewaschenen und gereinigten Guttapercha, dann hat man wieder gar keinen Anhnltspunkt für das Rohmaterial, für welches man bezahit. AuBerden bängt dann im gewaschenen Material die Viskusitat von der
Behandlungsweise bein Waschen und Reinigen $a b$, indem die gewaschene Guttapercha durch den Wasch- und Reinigungsprozefi stark depolymerisiert wird. Der Praktiker hat also nicht viel oder eigentlich gar nichts von der Viskositätsbestimmung.

Den Erweichungspunkt hingegen einer Guttaperchasorte zu kcnnen, ist für den Praktiker von groker Bedeutung, da or haufig vor die Aufgabe gestellt wird, Isoliermassen von ganz bestimmtem Erweichungspunkt herzustellen.

$\mathrm{Da}$ die Guttaperchaharze verseifbar sind, spielen auch die Harzbestimmungen für den Praktiker für die Herstellung von Mischungen eine große Rolle. Vom Wassergehalte hängt selbstverständlich die Leitfähigkeit der Mischungen ab. Nachdem die meiste Guttapercha zur Kabelisolierung Verwendung findet, erklärt sich hieraus die Bedeutung der Wasserbestimmungen.

\section{Welchen praktischen Wert haben mechanische Prüfungen des Kauts huks? \\ Elac kolloidchemische Unterouchung.}

Von Dr. Rudolf Ditmar (Graz).

(Eingezangen am 15. April 1912)

Eine Ablandlung von E. H. F r öl i c h'): „Beiträge zur mechanischen Prüfung von Kautschukwaren“, gibt mir Veranlassung zur Diskussion der Frage, welchen praktischen Wert mechanische Prüfungen des Kautschuks haben. Gleichzeitig möchte ich bemerken, dab die nachfolgenden Ueberlegungen mein Kautschuk-Glauhensbekenntnis darstellen, zu dem ich mich leider nach neunjähriger praktischer und theoretischer Tätigkeit durchringen mubte.

E. H. Frölich beobachtete sehr richtig, daB es sehr viele Faktoren sind, die den chemischen und physikalischen Zustand einer Weichgummiware, also die Eigenschaften der Ware, bedingen. Er unterscheidet die Fabrikation in zwei Hauptteile, die Herstellung der Mischung und die Vulkanisation des Artikels. Jeder davon zerfällt wieder in eine Anzahl Unterstufen:

1. Kautschuk.

$$
\text { A. Mischung. }
$$

a) Quantität desselben,

b) Kautschuksorte, (1912). c) physikalischer Zustand, abhängig von der Bearbeitung auf der Walze.

2. Zusătze.

a) Quantitat derselben,

b) Qualität derselben,

c) Art derselben.

3. Eventuelle nachträgliche Bearbeitung der Mischung auf der Schlaurchmaschine oder sonstigen den Kautschuk angreifenden Apparaten.

B. Vulkanisation.

1. HeiBvulkanisation.

a) Schwefelmenge,

b) Temperatur,

c) Zeitdauer,

d) Wärmeaufnahmefähigkeit der Masse, abhängig von Dimension derselben und Qualität der Mischung,

e) Wärmedurchlässigkeit der direkten Umgebung, ob frei geheizt oder in Talkum oder in Eisenform u. dgl. mehr,

f) Wärmeübertragungsfähigkeit des Mediums, ob Dampf, heiBe Luft oder Wasser, 